Journal of Mathematics and Informatics

Vol. 7, 2017, 73-78

ISSN: 2349-0632 (P), 2349-0640 (online)

Published 9 May 2017

www.researchmathsci.org

DOI: http://dx.doi.org/10.22457/jmi.v7a9

Journal of

Mathematics and

Informatics

\title{
A Survey of Stock Forecasting Model Based on Artificial
}

\section{Intelligence Algorithm}

\author{
Jia-Xuan Deng and Gui Gan \\ Department of Information and Computing Science \\ Chongqing University of Posts and Telecommunications \\ Nanan, 400065, Chongqing, P.R.China. E-mail: 3091600852@qq.com \\ Received 18 April 2017; accepted 5 May 2017
}

\begin{abstract}
Stock forecasting technology has become a hot spot for scholars and investors, with its unique charm to attract a large number of scholars and investors to be committed to the stock market forecast research. Particularly, intelligent forecasting technology has made significant achievements in this regard. This paper describes 14 advanced neural networks and support vector machine forecasting techniques at home and abroad, analyzes and summarizes the characteristics and key points of each forecasting method. Finally, it puts forward the existing problems of stock forecasting research methods and explores the prospect of the future.
\end{abstract}

Keywords: stock forecasting, neural network, support vector machine

AMS Mathematics Subject Classification (2010): $62 M 20$

\section{Introduction}

The stock market is one of the important components of the national economy and the most important way for the company to raise funds. Not only will investors and ordinary people be regarded as investment tools, but also predicting the future value of the stock market is the right decision whether to buy or sell shares, as the stock market a substantial impact on individuals' and nationals' economy. But because of so many factors such as economic, politics, environment and other factors determining the parameters, it is difficult to predict the stock price movements. For many years, the traditional statistical prediction methods are linear regression, time series analysis, chaos dynamics prediction method. However, due to the uncertainty of the stock market, these methods are failed or partially successful. With the rapid development of artificial intelligence, researchers have been devoted to the stock forecasting, bringing a lot of new 
Jia-Xuan Deng and Gui Gan

technologies and new methods.

\section{Neural network prediction technology}

Neural network technology can imitate the human's brain to do intelligent processing, with adaptive function for messy information, non-deterministic law, which make it quickly become a stock research hotspot.

Li Han et al. ${ }^{[3]}$ (2016) derived the LM (Levenberg-Marquardt) -BP neural network prediction method, design and develop its calculation program. They used the difference between the actual value and the predicted value as the anti-error to adjust the weight of the neural network by layer and test the model's stock price forecasting ability. Motion Billah et al. ${ }^{[4]}$ (2016) combined the LM algorithm with the adaptive neural network fuzzy reasoning system (ANFIS), which reduced the error and had higher accuracy than ANFIS and traditional LM algorithms. In addition, Zhang Wei et al. ${ }^{[5]}$ (2015) introduced the clustering analysis preprocessing data to improve the crossover operator and the mutation operator of the adaptive genetic algorithm, establishing a model of rough set attribute reduction and neural network based on this genetic algorithm. Xiaona Qi et al. ${ }^{[6]}$ (2016) combined the LM algorithm, the genetic algorithm and the traditional BP algorithm to establish the GA-LMBP neural network prediction model.

Sun Chen et al. ${ }^{[7]}$ (2016) used the cuckoo algorithm to optimize the neural network (CS-BP) to predict the stock price. They converted the closing price into some representative technical indicators (closing price, volume, PSY, etc.). Compared with PSO-BP model and GA-BP model, it was found that CS-BP model was superior to the other two algorithms, which could effectively predict the stock market.

Zhaoyue Hu et al. ${ }^{[8]}$ (2016) applied the additional momentum method to find the initial weights and thresholds of the BP neural network and applied the principal component analysis to extract the principal component with the contribution rate of $90 \%$ as the input variable to establish the PCA-AM-BP model. The model reduced the average error of the prediction, while the running time was greatly reduced and the convergence rate of the algorithm was accelerated.

Sun et al. ${ }^{[9]}$ (2016) decomposed the original non-stationary time series into time series components at different scales by wavelet decomposition and reconstruction. Elman neural network and BP neural network model were used according to their respective characteristics. Coefficients were modeled and predicted. The predicted values were obtained by integrating the coefficients of each layer. Compared with the traditional neural network prediction method, this method not only improves the prediction accuracy obviously, but also more in line with the actual ups and downs of the stock.

Chijie Lu et al. ${ }^{[10]}$ (2011) used high quantization resolution and large generalization size to reduce generalization errors and used efficient and fast hash codes to accelerate 
A Survey of Stock Forecasting Model Based on Artificial Intelligence Algorithm many-to-many mapping, establishing an efficient Cerebellar model joint control neural network (CAMC NN). Based on the experimental results, it was found that the performance of CMAC NN scheme was superior to robustness evaluation and support vector regression (SVR) and back propagation neural network (BPNN).

In contrast to the above-mentioned researchers exploring the algorithm of optimizing the neural network, Yixuan Li et al. ${ }^{[11]}$ (2015) used the neural network to study the influence of investor sentiment on stock price, verifying whether the emotion of microblogging emotion and traditional market survey data was related to the stock market. Based on the nonlinear BP neural network, they established the forecasting model and through the simulation, found that the domestic microblogging platform data on the stock market had a certain representation and influence, with better predictive ability than market data.

\section{Support vector machine (SVM) prediction technology}

Support Vector Machine (SVM) is a new generation of machine learning technology based on statistical learning theory and developed by Va Pnik, which can solve the practical problems such as small sample, non-linearity, high dimension and local minima. It has been successfully applied to classification, Regression and time series prediction. Compared with the neural network, it has greater advantages, such as the global optimal solution, better generalization ability, easy to determine the structure and so on.

Gangmol Jaiwang et al. ${ }^{[12]}$ (2016) improved the prediction accuracy by comparing the four kernel functions of SVM and used the "majority voting" technique and attribute selection algorithm to create high-precision prediction SVM model.

Guisheng Zhang et al. ${ }^{[13]}$ (2015) made use of the advantage of SVM's dealing with high - dimensional nonlinear data to overcome the shortcomings of traditional linear model analysis and processing non-linearity of yield data, proposing a new SVM-GAPCH prediction model based on the proximity information feature selection. The model were superior to the ARMA-GARCH traditional model on denoising the timing data, trend discrimination and prediction accuracy.

Chen X et al. ${ }^{[14]}$ (2015) used PLR to establish the stock trading signal prediction SVM model, in which PLR was a good way to extract valuable information from the time series and check the turning point in the study. The results show that SVM was better than BPN Generalization ability.

Kun $\mathrm{Li}$ et al. ${ }^{[15]}$ combined the wavelet theory with the support vector machine method to introduce the wavelet basis function to construct the kernel function of the support vector machine, obtaining a stock forecasting model with wavelet support vector machine regression, which overcame the local optimal problem of neural network, excluding the effects of artificial noise. 
Jia-Xuan Deng and Gui Gan

\section{Combination forecasting technology combined with ANN and SVM}

With the development of various prediction theories and technologies, there are more and more methods for stock market forecasting, with advantages and disadvantages of various methods exist. It is difficult for the forecasters to choose the best forecasting method. Therefore, a combination forecasting method is proposed, which combines two or more different prediction methods to predict the same prediction object. This method combines the advantages of a single prediction method and improves the prediction accuracy to a certain extent. Next, we will propose a new type of stock forecasting technology combined by neural network and support vector machine method.

Oztekin A et al. ${ }^{[16]}$ (2016) developed a generic methodology to predict daily stock price movements by deploying and integrating three data analytical prediction models: adaptive neuro-fuzzy inference systems, artificial neural net- works and support vector machines. Using a ten-fold stratified cross-validation to minimize the bias of random sampling, this study demonstrates that the support vector machine outperforms the other models. For all three predictive models, accuracy in predicting down movements in the index outweighed accuracy in predicting the up movements. The study yielded more accurate forecasts with fewer input factors compared to prior studies of forecasts for securities trading on Borsa Istanbul. This effective data analysis method could also be easily applied to other emerging market stock return series.

In addition to the above-mentioned combination forecasting method, we can also consider the use of time series, wavelet analysis, fuzzy technology and genetic algorithm and support vector machine to improve the stock forecast accuracy. Among them, Zhang combined the time series and neural network for stock forecasting, and achieved good results. Pai et al. combined the time series and support vector machine method to forecast the stock price, and the prediction effect was better than the single method [18].

\section{Conclusion}

The use of artificial intelligence to study the stock market with feedback nonlinear system is one of the hotspots in finance. In view of this hot spot, this paper studies the current advanced artificial intelligence stock forecasting model, classifies them and finds that there are different advantages and disadvantages of any model. Among them, the need to further explore the following issues:

1. For the neural network correlation models, because the neural network learning algorithm uses the principle of empirical risk minimization, lacks quantitative analysis and theoretical results of complete mechanism, and takes all reasoning into a numerical calculation, the information must be lost, even if use other algorithms to optimize it. This leads to the lack of neural network promotion ability, high network 
A Survey of Stock Forecasting Model Based on Artificial Intelligence Algorithm training accuracy and poor prediction accuracy.

2. SVM-related prediction models accuracy is not high, so there is a large number of real price inflection point which cannot be predicted.

3. Most of the models can only successfully predict the short-term stock price, but cannot judge the long-term stock price effectively. This success contains too many occasional factors. Only by constantly incorporating new data into the model and achieving rolling predictions can we provide a more rigorous test of the universality of the model.

4. The purpose of stock price forecast is to find a pattern that can be profitable with great probability, which means that the forecasting model should achieve the capture of the unusual moving points. In other words, find the quantitative characteristics when the stock price changes (such as a substantial surge). However, most of the models cannot find these points, which is also the direction that can be studied in the future.

5. Although a number of artificial intelligence models are introduced, there is no standard or rule of thumb to determine which artificial intelligence model is more suitable for stock forecasting.

\section{REFERENCES}

1. L.Guoping, Y.Guangqing and C.Senfa, A summary of gray forecasting research on China 's stock price, Journal of Southeast University, 7(2) (2005) 28-30.

2. W.Hao, A summary of stock market price forecasting models in China, Journal of Chengdu Normal University, 25(7) (2009) 58-60.

3. H.Li, Research on stock forecasting based on LM-BP neural network, Northeast Agricultural University, 2016.

4. M.Billah, S.Waheed and A.Hanifa, Stock market prediction using an improved training algorithm of neural network, Rajshahi, Bangladesh: Institute of Electrical and Electronics Engineers Inc., 2016.

5. Z.Wei, F.Nianbo and W.Wenjia, Research on stock forecasting model based on adaptive genetic algorithm, Computer Engineering and Applications, 51(4) (2015) 254-259.

6. Q.Xiaona and C.Weigang, Stock forecasting based on genetic LMBP neural network model, Wireless Internet Technology, (3) (2016) 86-87.

7. S.Chen, L.Yang, L.Xiaoge, et al., Price forecasting based on optimized bp neural network model based on cuckoo algorithm, Computer Applications and Software, (2) (2016) 276-279.

8. H.Zhaoyue and B.Yanping, Stock forecasting based on improved BP neural network, Economic and Trade Practice, (2) (2016) 276. 
Jia-Xuan Deng and Gui Gan

9. S.Bingjie, T.Rui, Z.Yi, et al., Research on neural network stock forecasting based on wavelet analysis, Computer and Digital Engineering, 44(6) (2016) 1031-1034.

10. C.-J.Lu, J.-Y.Wu, An efficient CMAC neural network for stock index forecasting, Expert Systems with Applications, 38(12) (2011) 15194-15201.

11. L.Yixuan, Research on stock forecast based on microblogging emotion, Foreign Economic and Trade University, 2015, (4) (2015) 24-42.

12. G.Jaiwang and P.Jeatrakul, A forecast model for stock trading using support vector machine, Chiang Mai, Thailand: Institute of Electrical and Electronics Engineers Inc., 2016.

13. Z.Guisheng and Z.Xindong, Research on SVM-GARCH stock price forecasting model based on neighbor mutual information, China Management Science, 24(9) (2016) 11-20.

14. X.Chen and Z.J.He, Prediction of stock trading signal based on support vector machine, International Conference on Intelligent Computation Technology and Automation. IEEE, (2015) 651-654.

15. L.Kun and T.Mengyu, Stock forecasting based on wavelet support vector machine regression, Statistics and Decision, (6) (2014) 32-36.

16. A.Oztekin, R.Kizilaslan, S.Freund, et al., A data analytic approach to forecasting daily stock returns in an emerging market, European Journal of Operational Research, 253(3) (2016) 697-710.

17. Z.Yalei, A survey of stock market forecasting based on neural network, Management Manager, 9X (2013) 114-114.

18. C.Qiao, L.Lili and X.Lijuan, Research progress of stock forecast, Monthly Business Culture, (6) (2012) 313-314. 